\title{
Industry concern over EU hepatotoxicity guidance
}

Recent draft guidance highlights the challenges that industry and regulators face to predict rare drug-induced liver injury.

\section{Bethan Hughes}

At the end of August 2008, the consultation period ended for comments on the European Medicines Agency's (EMEA's) draft non-clinical guidance on drug-induced hepatotoxicity. The agency identified a need for this guidance following critical assessment of cases of hepatotoxicity that led to post-market withdrawal of approved drugs (http://www.emea.europa.eu/pdfs/human/ swp/15011506en.pdf). “The non-clinical data available at the time of the marketing application contained signals that might have predicted that hepatotoxicity," says Jean-Marc Vidal of the EMEA's Safety and Efficacy of Human Medicines Sector, London, UK.

Now the EMEA aims to establish a harmonized approach to identify and interpret hepatotoxic signals. "By providing a better prediction of the hepatotoxic potential of developing drugs and a better understanding of its human relevance, later hepatic adverse reactions would be expected to be reduced," says Beatriz Silva Lima, Chair of the EMEA's Safety Working Party. No timeline is available for the final guidance because, says Silva Lima, "important discussions are still ongoing."

While agreeing that there is an urgent need to better understand non-clinical signs of drug-induced liver injury (DILI), the European Federation of Pharmaceutical Industries and Associations (EFPIA) finds it premature to issue a specific regulatory guidance. "At this time point, not all models mentioned in the draft guidance are accepted as fit for use to screen our compounds," says Steven Spanhaak, Chair of the EFPIA safety ad-hoc working group and principal scientist in toxicology at Johnson \& Johnson PRD, Belgium.

One overarching concern, explains Spanhaak, is the retrospective assessment that the non-clinical data contained signals that could have predicted DILI. The draft guidance fails to indicate whether or not similar signals could also be identified for compounds that have not shown DILI in humans and thus Spanhaak thinks that industry would be presented with many false-positive signals that would require additional preclinical in vitro and in vivo studies without providing additional relevant safety information. He says, "We have shared our comments with EMEA and are confident that they will be taken into account. We agree with the overall objective, but still need to discuss the various aspects related to this complex issue."

Another issue is that preclinical studies do not always identify the potential for DILI. A recent study published by an industry-led initiative known as the Safety Intelligence Program (SIP) Board (http://www.biowisdom. com/files/SIP Board Species_Concordance. pdf) quantifies this using a collection of extracted data evidenced in both Medline abstracts and the EMEA European Public Assessment Reports showing that $38-51 \%$ of DILI in humans was not detected in preclinical tests. The SIP Board is now working with other communities to try to understand why the human hepatotoxic potential of these compounds could not be predicted.

In contrast to the EMEA, rather than trying to provide non-clinical guidance on DILI, the FDA issued draft guidance on how to assess the potential for a drug to cause severe hepatic injury during clinical development (http:// www.fda.gov/Cder/guidance/7507dft.pdf). "The FDA gives guidance for decision-making based on biomarkers without necessarily stopping the study or development of the compound," says Jack Reynolds, Chair of the Drug Safety Executive Council, Massachusetts, USA. Reynolds thinks that the EMEA's guidance on preclinical studies does not help tackle our lack of knowledge of rare hepatotoxic events. "The gap is in understanding human hepatic injury," he says.

The research community are acutely aware of this knowledge gap and in recent years initiatives have started to address the problem. One example is the DILI Network established by the US National Institutes of Health. Since January 2004, the DILI Network has been creating a genomic DNA registry of patients who have experienced idiosyncratic DILI. "We are now entering the analysis phase," says Paul Watkins, Chair of the DILI Network steering committee and Director of the Hamner Center for Drug Safety Sciences,
University of North Carolina at Chapel Hill, USA, who expects it to take 5-10 years to understand the relationship between genotype and phenotype in these patients. Also identifying DNA variants in patients who have experienced severe DILI is the International Serious Adverse Event Consortium (http:// www.saeconsortium.org), launched in August 2007, which is expected to complete phase I of its research plan in October 2009.

These initiatives, however, study the effects of a limited number of drugs that cause hepatotoxicity. "Part of what is missing, and what might be more fruitful for regulators to facilitate, are broad approaches to study the liver as an organ of injury," says Reynolds. One such approach could be through the FDA's Critical Path collaboration with companies such as Entelos (California, USA) to develop a virtual model of human DILI.

While the research community increases its understanding of severe DILI, there remains a need to improve the predictability of hepatotoxicity during drug development. For example, the FDA aims to increase the number of non-clinical biomarkers of liver safety through the Predictive Safety Testing Consortium - a public-private partnership led by the non-profit Critical Path Institute.

"Several efforts are [also] ongoing in the EU," says Spanhaak, "one is InnoMed PredTox [a joint industry and EU collaboration looking at what combination of 'omics' technologies deliver the best predictive results for hepatotoxicity and/or nephrotoxicity; http:// www.innomed-predtox.com] with a follow up in the Innovative Medicines Initiative (IMI) which will begin later this year [http:// imi.europa.eu/calls-01 en.html]. Another important project amongst the IMI first call topics is on in silico toxicity prediction."

In part, the IMI in silico prediction call will aim to address one of the biggest gaps in drug safety knowledge - that there is no single database with historical proprietary safety data for companies to predict the effect of a chemical structure based on what is already known. "Companies will be able to compare their chemical structures with the database and make a prediction of hepatotoxicity or nephrotoxicity," says Spanhaak. To protect proprietary information companies cannot have access to structures that do not belong to them, but that will not prevent the prediction of liability.

"This is a first step towards a shared, across company safety database," says Spanhaak, "we need to move forward in this area and be more open to share the safety data to the benefit of all compounds in development. There would be more to gain than to lose by this perspective." 